HU-EP-09/52

DESY 09-179

\title{
Upper and lower Higgs boson mass bounds from a lattice Higgs-Yukawa model with dynamical overlap fermions
}

\section{Philipp Gerhold*}

Institut für Physik, Humboldt-Universität zu Berlin, 12489 Berlin, Germany

E-mail: gerhold@physik.hu-berlin.de

\section{Karl Jansen}

DESY, 15738 Zeuthen, Germany

E-mail: Karl. Jansen@desy.de

\begin{abstract}
We study a lattice Higgs-Yukawa model emulating the same Higgs-fermion coupling structure as in the Higgs sector of the electroweak Standard Model, in particular, obeying a GinspargWilson version of the underlying $\mathrm{SU}(2)_{L} \times \mathrm{U}(1)_{Y}$ symmetry, being a global symmetry here due to the neglection of gauge fields in this model. In this paper we present our results on the cutoffdependent upper Higgs boson mass bound at several selected values of the cutoff parameter $\Lambda$.
\end{abstract}

The XXVII International Symposium on Lattice Field Theory

July 26-31, 2009

Peking University, Beijing, China

\footnotetext{
*Speaker.
} 


\section{Introduction}

With the existing evidence for the triviality of the Higgs sector of the electroweak Standard Model, rendering the removal of the cutoff $\Lambda$ from the theory impossible, physical quantities in this sector will, in general, depend on the cutoff. Though this restriction strongly limits the predictive power of any calculation performed in the Higgs sector, it opens up the possibility of drawing conclusions on the energy scale $\Lambda$ at which new physics has to set in, once, for example, the Higgs boson mass has been determined experimentally.

The main target of lattice studies of the Higgs-Yukawa sector of the electroweak Standard Model has therefore been the non-perturbative determination of the cutoff-dependence of the upper and lower bounds of the Higgs boson mass $[1,2]$ as well as its decay properties. There are two main developments which warrant the reconsideration of these questions. First, with the advent of the LHC, we are to expect that properties of the Standard Model Higgs boson, such as the mass and the decay width, will be revealed experimentally. Second, there is, in contrast to the situation of earlier investigations of lattice Higgs-Yukawa models $[3,4,5,6]$, a consistent formulation of a Higgs-Yukawa model with an exact lattice chiral symmetry [7] based on the Ginsparg-Wilson relation [8], which allows to emulate the chiral character of the Higgs-fermion coupling structure of the Standard Model on the lattice while lifting the unwanted fermion doublers at the same time.

Since the question for the lower Higgs boson mass bound as well as the phase structure of the underlying model has already been addressed in Refs. [9, 10, 11, 12], we will here focus only on the discussion of our results concerning the cutoff-dependent upper Higgs boson mass bound.

\section{The $\mathbf{S U}(2)_{L} \times \mathbf{U}(1)_{Y}$ lattice Higgs-Yukawa model}

The model we consider here is a four-dimensional lattice Higgs-Yukawa model with a global $S U(2)_{L} \times U(1)_{Y}$ symmetry [7], aiming at the implementation of the chiral Higgs-fermion coupling structure of the pure Higgs-Yukawa sector of the Standard Model reading

$$
L_{Y}=y_{b}(\bar{t}, \bar{b})_{L} \varphi b_{R}+y_{t}(\bar{t}, \bar{b})_{L} \tilde{\varphi} t_{R}+c . c
$$

with $y_{t, b}$ denoting the top and bottom Yukawa coupling constants. Here we have restricted ourselves to the consideration of the top-bottom doublet $(t, b)$ interacting with the complex scalar doublet $\varphi$ ( $\tilde{\varphi}=i \tau_{2} \varphi^{*}, \tau_{i}$ : Pauli-matrices), since the dynamics of $\varphi$, containing the Higgs mode, is dominated by the coupling to the heaviest fermions. For the same reason we also neglect any gauge fields in this approach.

The fields considered in this model are thus the aforementioned doublet $\varphi$ as well as $N_{f}$ topbottom doublets represented by eight-component spinors $\bar{\psi}^{(i)} \equiv\left(\bar{t}^{(i)}, \bar{b}^{(i)}\right), i=1, \ldots, N_{f}$. The chiral character of the targeted coupling structure (2.1) can then be preserved on the lattice by constructing the fermionic action $S_{F}$ on the basis of the Neuberger overlap operator [13] according to

$$
S_{F}=\sum_{i=1}^{N_{f}} \bar{\psi}^{(i)} \mathscr{M} \psi^{(i)}, \quad \mathscr{M}=\mathscr{D}^{(o v)}+P_{+} \phi^{\dagger} \operatorname{diag}\left(y_{t}, y_{b}\right) \hat{P}_{+}+P_{-} \operatorname{diag}\left(y_{t}, y_{b}\right) \phi \hat{P}_{-},
$$

where the scalar field $\varphi_{x}$ has been rewritten as a quaternionic, $2 \times 2$ matrix $\phi_{x}^{\dagger}=\left(\tilde{\varphi}_{x}, \varphi_{x}\right)$, with $x$ denoting the site index of the $L_{s}^{3} \times L_{t}$-lattice. The left- and right-handed projection operators $P_{ \pm}$ 
and the modified projectors $\hat{P}_{ \pm}$are given as

$$
P_{ \pm}=\frac{1 \pm \gamma_{5}}{2}, \quad \hat{P}_{ \pm}=\frac{1 \pm \hat{\gamma}_{5}}{2}, \quad \hat{\gamma}_{5}=\gamma_{5}\left(\mathbb{1}-\frac{1}{\rho} \mathscr{D}^{(o v)}\right)
$$

with $\rho$ being the radius of the circle of eigenvalues in the complex plane of the free Neuberger overlap operator [13].

This action now obeys an exact global $\mathrm{SU}(2)_{L} \times \mathrm{U}(1)_{Y}$ lattice chiral symmetry. For $\Omega_{L} \in$ $\mathrm{SU}(2)$ and $\varepsilon \in \mathbb{R}$ the action is invariant under the transformation

$$
\begin{aligned}
\psi \rightarrow U_{Y} \hat{P}_{+} \psi+U_{Y} \Omega_{L} \hat{P}_{-} \psi, & \bar{\psi} \rightarrow \bar{\psi} P_{+} \Omega_{L}^{\dagger} U_{Y}^{\dagger}+\bar{\psi} P_{-} U_{Y}^{\dagger}, \\
\phi \rightarrow U_{Y} \phi \Omega_{L}^{\dagger}, & \phi^{\dagger} \rightarrow \Omega_{L} \phi^{\dagger} U_{Y}^{\dagger}
\end{aligned}
$$

with the compact notation $U_{Y} \equiv \exp (i \varepsilon Y)$ denoting the respective representations of the global hypercharge symmetry group $U(1)_{Y}$ for the respective field it is acting on. In the continuum limit Eq. (2.4-2.5) eventually recover the (here global) continuum $\mathrm{SU}(2)_{L} \times \mathrm{U}(1)_{Y}$ chiral symmetry.

Finally, the purely bosonic part $S_{\varphi}$ of the total lattice action $S=S_{F}+S_{\varphi}$ is given by the usual lattice $\Phi^{4}$-action

$$
S_{\varphi}=\sum_{x}\left\{\frac{1}{2} \nabla_{\mu}^{f} \varphi_{x}^{\dagger} \nabla_{\mu}^{f} \varphi_{x}+\frac{1}{2} m_{0}^{2} \varphi_{x}^{\dagger} \varphi_{x}+\lambda\left(\varphi_{x}^{\dagger} \varphi_{x}\right)^{2}\right\}
$$

with the bare mass $m_{0}$, the forward derivative operator $\nabla_{\mu}^{f}$ in direction $\mu$, and the bare quartic coupling constant $\lambda$.

\section{Upper Higgs boson mass bounds}

In the following the aim will be to determine the largest Higgs boson mass attainable in the considered Higgs-Yukawa model for a given cutoff $\Lambda$, while being in consistency with phenomenology. Here, the later requirement is translated into three matching conditions fixing the vacuum expectation value $v$ of the scalar field $\varphi$ as well as the top and bottom quark masses, according to

$$
246 \mathrm{GeV}=\frac{v_{r}}{a} \equiv \frac{v}{\sqrt{Z_{G}} \cdot a}, \quad \Lambda=a^{-1}, \quad y_{t, b}=\frac{m_{t, b}}{v_{r}}
$$

where we restrict ourselves to the mass degenerate case with $m_{t} / a=m_{b} / a=175 \mathrm{GeV}$ in order to guarantee the fermion determinant $\operatorname{det}(\mathscr{M})$ to be real. As a starting point, we simply use the tree-level relations in Eq. (3.1) to fix the bare Yukawa coupling constants. The actually resulting fermion masses have explicitly been computed on the lattice, which would eventually allow for a more precise tuning of the Yukawa coupling constants beyond the tree-level relation in some follow-up studies. However, though not explicitly demonstrated in this paper, it is found that the Yukawa coupling constants determined by Eq. (3.1) already reproduce the targeted fermion masses with a deviation smaller than $2 \%$ in the here considered parameter setups. The cutoff parameter $\Lambda$ can then (non-uniquely) be defined as the inverse lattice spacing $a^{-1}$, which is obtained by matching the lattice result on the renormalized vacuum expectation value $v_{r}=v / \sqrt{Z_{G}}$ with its phenomenological value. The underlying Goldstone renormalization constant $Z_{G}$ is given as

$$
Z_{G}^{-1}=\left.\frac{\mathrm{d}}{\mathrm{d} p_{c}^{2}}\left[\tilde{G}_{G}^{c}\left(p_{c}^{2}\right)\right]^{-1}\right|_{p_{c}^{2}=-m_{G}^{2}}, \quad \tilde{G}_{G}(p)=\frac{1}{3} \sum_{\alpha=1}^{3}\left\langle\tilde{g}_{p}^{\alpha} \tilde{g}_{-p}^{\alpha}\right\rangle, \quad \tilde{G}_{H}(p)=\left\langle\tilde{h}_{p} \tilde{h}_{-p}\right\rangle,
$$


with $\tilde{G}_{H, G}(p)$ denoting the lattice propagators of the Higgs and Goldstone modes in momentum space, respectively. For the details of how the aforementioned modes $\tilde{h}_{p}, \tilde{g}_{p}^{\alpha}$, and the vacuum expectation value $v$ are extracted from the scalar field $\varphi$, the interested reader is referred to Ref. [9]. The Goldstone mass $m_{G p}$ is then given by the pole of the Goldstone propagator, according to

$$
\left.\left[\tilde{G}_{G}^{c}\left(p_{c}^{2}\right)\right]^{-1}\right|_{p_{c}^{2}=-m_{G p}^{2}}=0,\left.\quad \operatorname{Re}\left(\left[\tilde{G}_{H}^{c}\left(p_{c}^{2}\right)\right]^{-1}\right)\right|_{p_{c}^{2}=-m_{H p}^{2}}=0 .
$$

Following the proposition in Ref. [14] the Higgs boson mass, on the other hand, is obtained here as the zero of the real part of the inverse Higgs propagator, being very close to the actual pole of the propagator [14] while being numerically much better accessible.

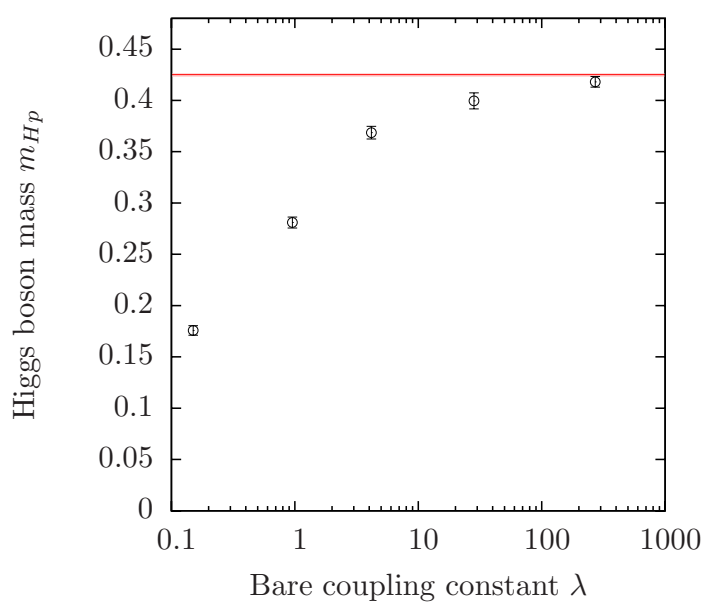

(a)

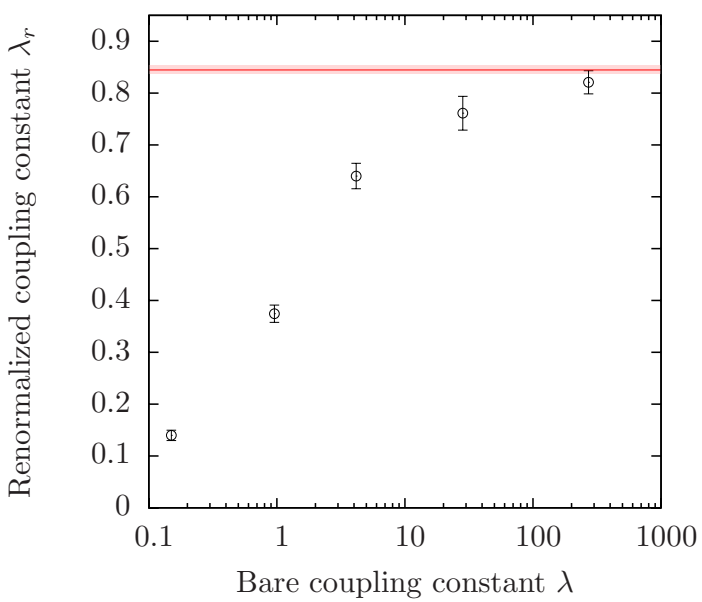

(b)

Figure 1: The Higgs boson mass $m_{H p}$ and the renormalized quartic coupling constant $\lambda_{r}$ are shown versus the bare coupling constant $\lambda$ in panels (a) and (b), respectively. These results have been obtained in direct Monte-Carlo calculations on a $16^{3} \times 32$-lattice with $N_{f}=1$. The cutoff parameter $\Lambda$ was intended to be kept constant, while the actually obtained values of $\Lambda$ fluctuate here between $1504 \mathrm{GeV}$ and $1549 \mathrm{GeV}$. The horizontal lines depict the corresponding results at $\lambda=\infty$, and the highlighted bands mark the associated statistical uncertainties.

For clarification it is remarked that $\tilde{G}_{H, G}^{c}\left(p_{c}\right)$ denote analytical continuations of the lattice propagators $\tilde{G}_{H, G}(p)$, since the latter are only defined at the discrete set of lattice momenta $p$. These analytical continuations have been obtained here by fitting the lattice propagators with fit functions derived from renormalized perturbation theory. As discussed in Ref. [14] the renormalized quartic coupling constant $\lambda_{r}$ can then be defined as

$$
\lambda_{r}=\frac{m_{H p}^{2}-m_{G p}^{2}}{8 v_{r}^{2}} .
$$

From perturbation theory one would expect the largest Higgs boson mass to be observed at infinite bare quartic coupling constant, i.e. $\lambda=\infty$. In Fig. 1 it is explicitly checked that the renormalized quartic coupling constant $\lambda_{r}$ as well as the Higgs boson mass $m_{H p}$ itself are indeed monotonic functions of the bare parameter $\lambda$, converging to their respective maximum at $\lambda=\infty$, as expected. For the purpose of determining the upper Higgs boson mass bound, the setting $\lambda=\infty$ is therefore adapted in the following. 
For the eventual determination of the cutoff-dependent Higgs boson mass bound $m_{H}^{u p}(\Lambda)$ several series of Monte-Carlo calculations have been performed at different values of $\Lambda$ and on different lattice volumes to finally allow for an infinite volume extrapolation. In order to tame finite volume effects as well as cutoff effects, we demand here as a minimal requirement that all particle masses $\hat{m}=m_{H p}, m_{t}, m_{b}$ in lattice units fulfill

$$
\hat{m}<0.5 \quad \text { and } \quad \hat{m} \cdot L_{s, t}>2,
$$

at least on the largest investigated lattice volumes. Assuming the Higgs boson mass to be below $700 \mathrm{GeV}$ this allows to reach energy scales between $1400 \mathrm{GeV}$ and $2800 \mathrm{GeV}$ on a $32^{4}$-lattice.

In the following we use $N_{f}=1, L_{t}=32$, and $L_{s}=12,16,20,24,32$ while the bare mass parameter $m_{0}$ is tuned to cover the aforementioned interval of accessible energy scales. In addition, corresponding lattice calculations have also been performed in the pure $\Phi^{4}$-theory, i.e. with $y_{t}=$ $y_{b}=0$, in order to estimate the strength of the fermionic contributions to the upper mass bound $m_{H}^{u p}(\Lambda)$. The obtained finite volume lattice data are presented in Fig. 2.

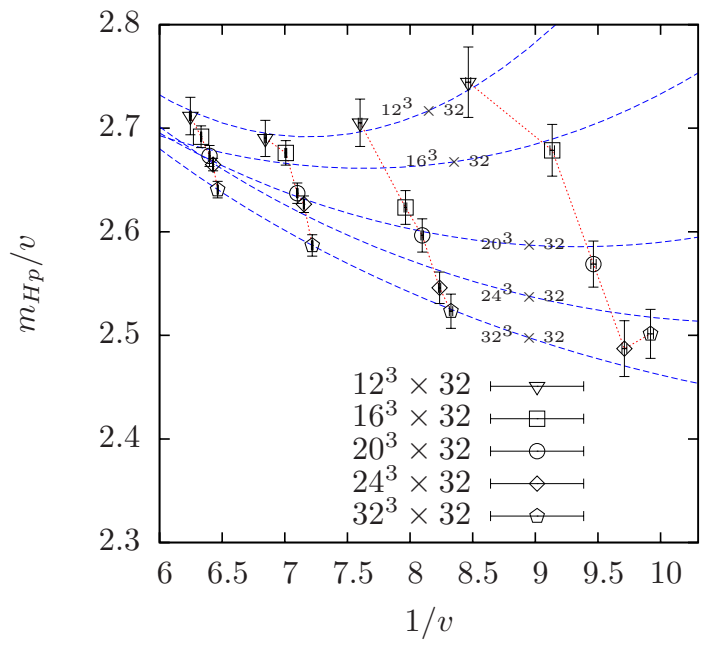

(a)

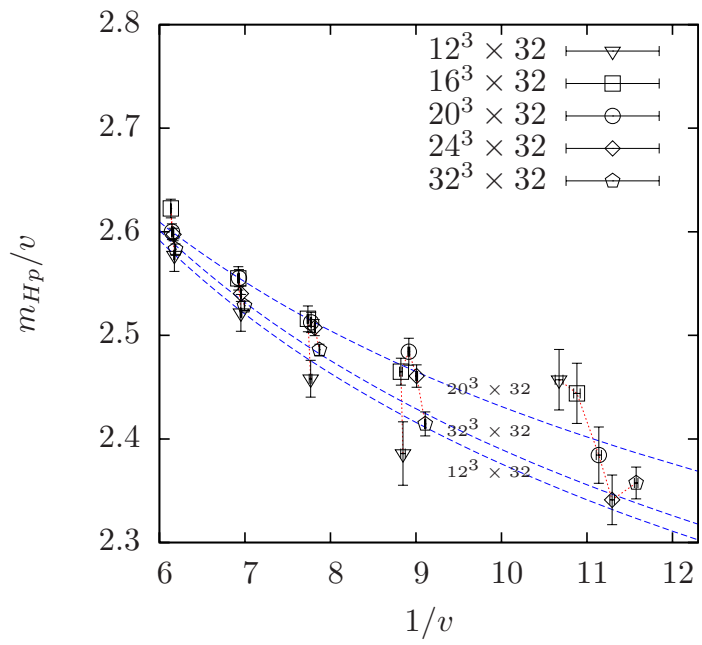

(b)

Figure 2: The Higgs propagator mass $m_{H p}$ is presented in units of the vacuum expectation value $v$ versus $1 / v$. Those Monte-Carlo results with identical parameter sets differing only in the underlying lattice volume are connected via dotted lines to illustrate the effects of the finite volume. The dashed curves depict the fits of the lattice results according to the finite size fit approach in Eq. (3.9) as explained in the main text. Panel (a) refers to the full Higgs-Yukawa model, while panel (b) shows the corresponding results of the pure $\Phi^{4}$-theory.

In order to understand the strong finite volume effects observed in Fig. 2a we consider here the constraint effective potential $U[\breve{v}]$. In Ref. [10] it has been derived for the degenerate case $y_{t}=y_{b}$ in the large $N_{f}$-limit with $\lambda \propto N_{f}^{-1}$ and $y_{t, b} \propto N_{f}^{-1 / 2}$. It then reads

$$
U[\breve{v}]=\frac{1}{2} m_{0}^{2} \breve{v}^{2}+\lambda \breve{v}^{4}+U_{F}[\breve{v}], \quad U_{F}[\breve{v}]=\frac{-4 N_{f}}{L_{s}^{3} \cdot L_{t}} \cdot \sum_{p} \log \left|v^{+}(p)+y_{t} \breve{v}\left(1-\frac{1}{2 \rho} v^{+}(p)\right)\right|^{2},
$$

where $U_{F}[\breve{v}]$ denotes here the fermionic contribution and $v^{+}(p)$ is the eigenvalue of the free overlap Dirac operator with non-negative imaginary part associated to the lattice momentum $p$. 


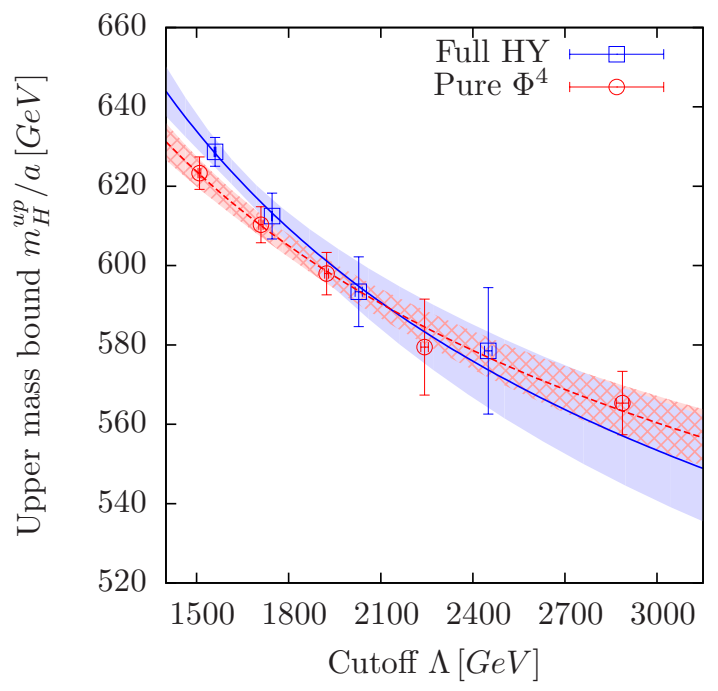

(a)

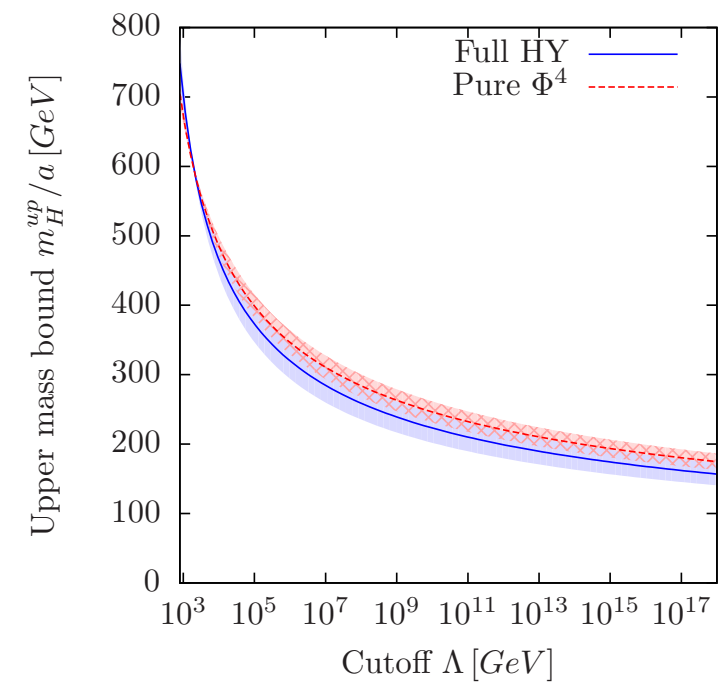

(b)

Figure 3: The cutoff dependence of the upper Higgs boson mass bound is presented in panel (a) as obtained from the infinite volume extrapolation results of the data in Fig. 2. The dashed and solid curves are fits of the data arising from the full Higgs-Yukawa model and the pure $\Phi^{4}$-theory, respectively, with the analytically expected cutoff dependence in Eq. (3.10). Panel (b) shows the latter fit curves extrapolated to larger values of the cutoff $\Lambda$. In both panels the highlighted bands reflect the uncertainty of the respective fit curves.

An estimate $m_{H e}$ of the Higgs boson mass can then be obtained from the curvature of the effective potential at its minimum, given by the vacuum expectation value $v$, yielding then

$$
m_{H e}^{2}=8 \lambda v^{2}-\left.\frac{1}{v} \frac{\mathrm{d}}{\mathrm{d} \breve{v}} U_{F}[\breve{v}]\right|_{\breve{v}=v}+\left.\frac{\mathrm{d}^{2}}{\mathrm{~d} \breve{v}^{2}} U_{F}[\breve{v}]\right|_{\breve{v}=v},
$$

which predicts the numerical results on $m_{H p}$ well in the weak coupling regime [9], i.e. for $\lambda \ll 1$. In this case, however, we consider the setting $\lambda=\infty$, rendering thus Eq. (3.7) inapplicable here. Replacing the bare parameter $\lambda$ with its renormalized counterpart $\lambda_{r}$, which is well justified at the considered order in $\lambda$, and exploiting also the expected functional form of the cutoff-dependence of $\lambda_{r}$, which has been derived in Ref. [14] according to

$$
\lambda_{r}=A_{\lambda} \cdot\left[\log \left(\Lambda^{2} / \mu^{2}\right)+B_{\lambda}\right]^{-1},
$$

where double-logarithmic terms have been neglected, $\mu$ denotes some unspecified scale, and $A_{\lambda} \equiv$ $A_{\lambda}(\mu), B_{\lambda} \equiv B_{\lambda}(\mu)$ are constants, one directly arrives at the expression

$$
m_{H e}^{2}=\frac{8 v^{2} A_{\lambda}}{\log \left(v^{-2}\right)+B_{\lambda}}-\left.\frac{1}{v} \frac{\mathrm{d}}{\mathrm{d} \breve{v}} U_{F}[\breve{v}]\right|_{\breve{v}=v}+\left.\frac{\mathrm{d}^{2}}{\mathrm{~d} \breve{v}^{2}} U_{F}[\breve{v}]\right|_{\breve{v}=v},
$$

which has been used to fit the finite volume lattice data in Fig. 2 with the free fit parameters $A_{\lambda}$, $B_{\lambda}$. From the good agreement between the analytical fit curves and the numerical data one learns that the finite size effects are well understandable already with the simple ansatz given in Eq. (3.9). In particular, the finite size effects in Fig. 2a, which are much stronger than in Fig. 2b, can mainly be ascribed to the fermionic contributions. This is also what one would have expected, since the top quark is the lightest particle in the here considered scenario. 
After having performed an infinite volume extrapolation of the finite size lattice data, the obtained results of that extrapolation are finally presented in Fig. 3a. These numerical data are moreover fitted with the analytically expected functional form of the cutoff-dependence of the Higgs boson mass, derived in Ref. [14] according to

$$
\frac{m_{H p}}{a}=A_{m} \cdot\left[\log \left(\Lambda^{2} / \mu^{2}\right)+B_{m}\right]^{-1 / 2},
$$

with $A_{m} \equiv A_{m}(\mu), B_{m} \equiv B_{m}(\mu)$ denoting the free fit parameters and $\mu$ being again some unspecified scale here. One learns from this presentation that the expected logarithmic decline of the Higgs boson mass with increasing cutoff parameter $\Lambda$ can very well be resolved. The fermionic contribution to the upper Higgs boson mass bound, however, can not clearly be identified with the here available statistics. Finally, it is tempting to extend the fit curves to very large values of $\Lambda$. This has been done in Fig. 3b. One finds that the resulting cutoff-dependent upper Higgs boson mass bound would reach a value around $160 \mathrm{GeV}$ at the Planck scale, which is in consistency with earlier perturbative studies within the given uncertainties.

\section{Acknowledgments}

We thank J. Kallarackal for discussions and M. Müller-Preussker for his continuous support. We are grateful to the "Deutsche Telekom Stiftung" for supporting this study by providing a Ph.D. scholarship for PG. We further acknowledge the support of the DFG through the DFG-project Mu932/4-1. The numerical computations have been performed on the HP XC400O System at the Scientific Supercomputing Center Karlsruhe and on the SGI system HLRN-II at the HLRN Supercomputing Service Berlin-Hannover.

\section{References}

[1] K. Holland and J. Kuti. Nucl. Phys. Proc. Suppl., 129:765-767, 2004.

[2] K. Holland. Nucl. Phys. Proc. Suppl., 140:155-161, 2005.

[3] J. Smit. Nucl. Phys. Proc. Suppl., 17:3-16, 1990.

[4] J. Shigemitsu. Nucl. Phys. Proc. Suppl., 20:515-527, 1991.

[5] M. F. L. Golterman. Nucl. Phys. Proc. Suppl., 20:528-541, 1991.

[6] A. K. De and J. Jersák. HLRZ Jülich, HLRZ 91-83, preprint edition, 1991.

[7] M. Lüscher. Phys. Lett., B428:342-345, 1998.

[8] P. H. Ginsparg and K. G. Wilson. Phys. Rev., D25:2649, 1982.

[9] P. Gerhold and K. Jansen. JHEP, 07:025, 2009.

[10] P. Gerhold and K. Jansen. JHEP, 09:041, 2007.

[11] P. Gerhold and K. Jansen. JHEP, 10:001, 2007.

[12] Z. Fodor, K. Holland, J. Kuti, D. Nogradi, and C. Schroeder. PoS, LAT2007:056, 2007.

[13] H. Neuberger. Phys. Lett., B427:353-355, 1998.

[14] M. Lüscher and P. Weisz. Nucl. Phys., B318:705, 1989. 